\title{
Nanoparticles for the induction of antigen-specific Tregs
}

\section{"...the generation of antigen-specific Tregs is viewed as a promising approach for the treatment of autoimmune disorders, but methods for generating significant numbers of antigen-specific Tregs in vivo or in vitro are still missing."}

\section{KEYWORDS: multiple sclerosis $\approx$ nanoparticles " therapy $\approx$ Tregs " Type 1 diabetes}

Specialized populations of Tregs control activity in healthy individuals. Treg deficits are usually associated with the development of autoimmune diseases. Thus, the expansion of antigen-specific Tregs is viewed as a potential therapeutic approach for autoimmune disorders; however, methods for generating significant numbers of antigenspecific Tregs in vivo or in vitro are still missing. Nanotechnology offers new tools for the expansion of antigen-specific Tregs and the treatment of autoimmune disorders. This article discusses recent examples of the use of nanotechnologybased approaches to re-establish immune tolerance and treat autoimmune diseases.

Autoimmune diseases result from the dysregulated reactivity of the immune system against self-antigens. In healthy individuals, the activity of the immune system is controlled by specialized populations of Tregs [1]. In patients afflicted by autoimmune diseases, however, Treg deficits are usually found [2]. Thus, the generation of antigen-specific Tregs is viewed as a promising approach for the treatment of autoimmune disorders, but methods for generating significant numbers of antigen-specific Tregs in vivo or in vitro are still missing.

Nanoparticles (NPs) have unique physical and chemical features that prompted their use in medicine. Some of these features are of particular interest for the field of drug delivery [3]:

- NPs can increase the solubility of drugs in vivo and protect them from enzymatic degradation;

- NPs can reduce the nonspecific binding and cell uptake of drugs in vivo, increasing their half-life;

- NPs can deliver drugs in a cell-type-specific manner, reducing off-target effects;

- Labeled NPs can be used to track the biodistribution of the drug in vivo.
Recent studies suggest that these and other features of NPs offer a new avenue for the induction of antigen-specific Tregs and the treatment of immune-mediated disorders.

\section{Expansion of memory-like CD8+ Tregs with NPs}

During the course of spontaneous autoimmune Type 1 diabetes in nonobese diabetic (NOD) mice, a population of low-avidity self-reactive $\mathrm{CD}^{+} \mathrm{T}$ cells acquires a regulatory phenotype. To expand these $\mathrm{CD} 8^{+}$Tregs, Santamaria and coworkers developed NPs containing complexes of disease-relevant self-peptides and the class I MHC (pMHC-NPs) [4].

The administration of pMHC-NPs abrogated the diabetogenic response and restored normoglycemia in diabetic NOD mice, and also in humanized NOD mice [4]. Mechanistic studies determined that pMHC-NPs were not taken up by $\mathrm{CD} 11 \mathrm{~b}^{+}, \mathrm{CD} 11 \mathrm{c}^{+}$or $\mathrm{B}$ cells, and instead targeted $\mathrm{T}$ cells in vivo, leading to the expansion of regulatory memory-like CCR7CXCR $3{ }^{+} \mathrm{CD} 62 \mathrm{~L}^{\mathrm{hi}} \mathrm{CD} 44^{\mathrm{hi}} \mathrm{CD} 122^{+} \mathrm{CD} 8^{+} \mathrm{T}$ cells, a phenotype that differs from that of 'classical' memory $\mathrm{T}$ cells. The $\mathrm{CD} 8^{+}$Tregs induced by pMHC-NPs produced IFN- $\gamma$, but did not proliferate or secrete IL-2 in response to stimulation. Moreover, these $\mathrm{CD} 8^{+}$Tregs showed suppressive activity in vitro and in vivo, as a result of their ability to kill APCs through a mechanism that involved IFN- $\gamma$, indoleamine 2,3-dioxygenase (IDO) and perforin. An important feature of the suppressive activity of the CD8 ${ }^{+}$Tregs induced by pMHC-NPs is that they suppressed the activation of $\mathrm{T}$ cells of different antigen specificities. Since several self-antigens are usually targeted during the course of autoimmune diseases [5] and the identity of all the antigens targeted by the autoimmune response in each individual patient is usually unknown, this bystander suppression

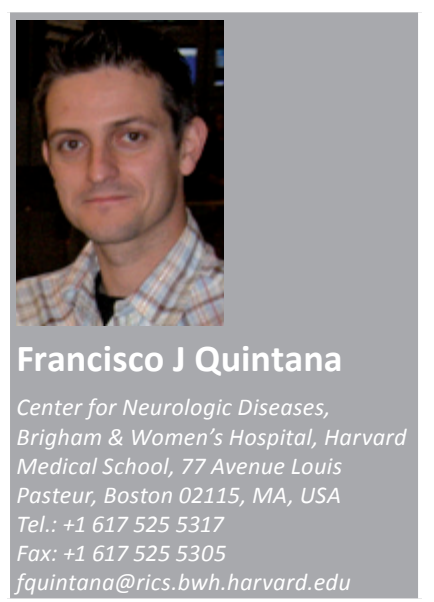

Future fSO $_{\text {part of }}$ 
is a desirable effect that increases the translational value of pMHC-NPs for the treatment of human Type 1 diabetes. However, a limitation of these pMHC-NPs is that in a clinical setting, it will require the generation of NPs loaded with peptide class I MHC matched to the HLA of each patient.

\section{Induction of antigen-specific tolerance with microparticles}

Antigen presentation by apoptotic APCs results in the induction of antigen-specific tolerance and Treg expansion [6]. Accordingly, the administration of soluble peptides cross-linked to syngeneic splenic leukocytes has been shown to treat several experimental autoimmune diseases [6]. In order to facilitate the clinical translation of these findings to the treatment of human autoimmune diseases, Miller and coworkers developed microparticles (MPs) to deliver self-antigens and exploit the immunoregulatory pathways triggered by apoptotic APCs [7].

\section{"Nanotechnology offers new opportunities for the induction of antigen-specific Tregs in vivo, without the limitations associated with the manipulation of patient cells in vitro."}

The administration of MPs loaded with myelin peptides ameliorated chronic relapsingremitting CNS autoimmunity in an experimental model of multiple sclerosis [7]. Following intravenous administration, the MPs were taken up by splenic macrophages in the marginal zone through a mechanism that involved MARCO, a scavenger receptor associated with the clearance of particulate debris. MPs suppressed the autoimmune response by several mechanisms, including abortive activation and the induction of anergy in pathogenic T cells, and the expansion of FoxP3 ${ }^{+}$Tregs that contributed to, but were not necessary for, these tolerogenic effects. The MPs developed by Getts et al. are not based on patient-matched peptide MHCs, facilitating their use in a clinical setup [7]. However, the mechanism of action of these MPs suggests that their tolerogenic effects only extend to the autoimmune response directed against the peptide epitopes included in the MPs. As already mentioned, the fine specificity of the autoimmune response differs between patients [8] and might even change in the same patient during the course of an autoimmune disease, thus the efficacy of therapies that do not promote bystander suppression is likely to be limited.

\section{Codelivery of tolerogenic aryl hydrocarbon receptor ligands \& self-antigens with NPs}

The ligand-activated transcription factor aryl hydrocarbon receptor (AHR) is an important regulator of the adaptive immune response [9]. Our group was among the first to describe the control of Th17, Tr1 and FoxP3 ${ }^{+}$Tregs by AHR in T cells [10-16]. Moreover, our group [14] and others [17-19] showed that the generation of Tregs by AHR ligands involves the induction of tolerogenic dendritic cells (DCs) that promote the differentiation of Tregs.

AHR has a promiscuous binding site that can interact with a broad array of synthetic and natural ligands. When inactive, AHR is located in the cytoplasm as part of a complex with other proteins, such as the $90-\mathrm{kDa}$ HSP90. Following interaction with a ligand, AHR translocates to the nucleus where it establishes protein-protein interactions with coactivators and additional transcription factors to control the transcriptional activity of target genes. Although the molecular mechanisms mediating the effects of AHR activation in DCs are mostly unknown, AHR has been shown to interact and modulate the activity of transcription factors known to play an important role in the regulation of DC activation, including AP1, NF- $\kappa B$, RAR and STATs [9].

Hauben et al. first demonstrated that AHR activation induces tolerogenic DCs that promote the differentiation and expansion of Tregs [17]. Subsequently, other groups confirmed these results and provided a mechanistic basis for these observations $[14,18,20]$. AHR activation results in the downregulation of MHC and costimulatory molecule expression in DCs [14]. Moreover, AHR activation limits the production of polarizing cytokines that promote the differentiation of Th1 and Th17 cells, interfering with the ability of DCs to promote the activation and differentiation of pathogenic T cells [14]. Finally, AHR activation triggers the expression of molecules that promote the differentiation of FoxP3 ${ }^{+}$Tregs $[14,18,19]$.

IDO catalyzes the degradation of tryptophan into anti-inflammatory kynurenines. Kynurenines promote the generation of FoxP $3^{+}$Tregs in the context of autoimmune disorders and during the immune response to tumors [21,22]. AHR activation induces IDO expression in DCs, consequently triggering the production of kynurenines, which promote the differentiation of functional FoxP3 ${ }^{+}$Tregs [18,19]. Notably, AHR expression in $\mathrm{T}$ cells is also needed for 
the differentiation of Foxp $3^{+}$Tregs triggered by kynurenine [18].

An additional mechanism used by AHR to promote Treg differentiation is the control of retinoic acid (RA) production by DCs. AHR activation upregulates the expression of enzymes involved in the synthesis of RA. RA produced by DCs boosts FoxP3 ${ }^{+}$Treg differentiation and it is thought to play an important role in the control of the immune response in mucosal tissues [23]. Indeed, blockade of RA signaling with specific inhibitors interferes with the differentiation of FoxP3 ${ }^{+}$Tregs triggered by AHR activation in DCs. Taken together, these data indicate that several mechanisms mediate the generation of FoxP3 ${ }^{+}$Tregs following AHR activation in DCs.

\section{"NPs loaded with ITE and myelin antigens promote the expansion of FoxP3 $3^{+}$Tregs that control the pathogenic $T$-cell response directed against a broad set of target self-epitopes that were not represented by the antigens included in the tolerogenic NPs."}

We found that the nontoxic AHR ligand 2-(1'H-indole-3'-carbonyl)-thiazole-4-carboxylic acid methyl ester (ITE) [14] induces tolerogenic DCs that expand the FoxP3 ${ }^{+}$Treg compartment and suppress the development of experimental autoimmune encephalomyelitis (EAE), an experimental model of multiple sclerosis. Based on the tolerogenic effects of AHR activation in DCs, we engineered NPs to co-deliver ITE and myelin antigens to APCs in vivo and induce CNS-specific Tregs [16]. The toxicity of most of the materials used to construct NPs is unknown [3], but colloidal gold has been used to treat rheumatoid arthritis for more than 50 years, showing no toxic or adverse effects [24]. Gold colloid NPs have large surface areas to conjugate molecules, which can be further stabilized by coating with polyethylene glycol [24]. Pegylated gold NPs show striking stability and excellent in vivo biodistribution and pharmacokinetic properties, and have been recently shown to be taken up by splenic DCs in vitro and in vivo. Thus, we used gold NPs to coadminister ITE and myelin antigens to APCs.

NPs increased the half-life and protected ITE from degradation by liver enzymes [16]. However, using an AHR-luciferase reporter cell line, we found that ITE contained in NPs was released and activated AHR signaling.
ITE-loaded NPs induced a tolerogenic phenotype in DCs in vitro and in vivo, characterized by a reduced ability to activate $T$ cells and promote their differentiation into Th1 and Th17 cells, concomitant with an increased ability to promote the differentiation of FoxP3 $3^{+}$Tregs. We found that NPs were taken up by DCs and activated AHR signaling in vivo [16]. NPs loaded with ITE and myelin antigens expanded the FoxP3 ${ }^{+}$Treg compartment and suppressed EAE, both in preventive and therapeutic paradigms. Moreover, the therapeutic effects of the NPs could be transferred with FoxP3 ${ }^{+}$Tregs and were also detected in chronic EAE models in which disease progression is driven by extensive intra- and inter-molecular epitope spreading. Taken together, these data suggest that NPs loaded with ITE and myelin antigens promote the expansion of FoxP3 ${ }^{+}$Tregs that control the pathogenic $\mathrm{T}$-cell response directed against a broad set of target self-epitopes that were not represented by the antigens included in the tolerogenic NPs.

\section{Conclusion}

The expansion of antigen-specific Tregs is considered a potential approach for the treatment of immune-mediated disorders. Nanotechnology offers new opportunities for the induction of antigen-specific Tregs in vivo, without the limitations associated with the manipulation of patient cells in vitro. Several factors, however, currently limit the clinical translation of NP-based approaches: the usually unknown long-term toxicity of the nanomaterials used, the difficulties linked to the production of homogeneous nanovaccine preparations on a large scale and the availability of nanovaccines that are not restricted by the MHC or antigen specificity of the autoimmune response in each patient. Once these limitations are overcome, tolerogenic NPs will certainly provide new therapeutic approaches for autoimmunity.

Financial \& competing interests disclosure

Research in FJ Quintana's laboratory is supported by grants AI075285 and AI093903 from the NIH, RG4111A1 from the National Multiple Sclerosis Society, and 17-2011-371 from the Juvenile Diabetes Research Foundation to FJ Quintana. The author has no other relevant affliations or financial involvement with any organization or entity with a financial interest in or financial conflict with the subject matter or materials discussed in the manuscript apart from those disclosed.

No writing assistance was utilized in the production of this manuscript. 


\section{References}

1 Sakaguchi S, Miyara M, Costantino CM, Hafler DA. FOXP3 ${ }^{+}$regulatory $\mathrm{T}$ cells in the human immune system. Nat. Rev. Immunol. 10(7), 490-500 (2010).

2 Cvetanovich GL, Hafler DA. Human regulatory $\mathrm{T}$ cells in autoimmune diseases. Curr. Opin. Immunol. 22(6), 753-760 (2010).

3 Sanvicens N, Marco MP. Multifunctional nanoparticles - properties and prospects for their use in human medicine. Trends Biotechnol. 26(8), 425-433 (2008).

4 Tsai S, Shameli A, Yamanouchi J et al. Reversal of autoimmunity by boosting memory-like autoregulatory T cells. Immunity $32(4), 568-580$ (2010).

5 Vanderlugt CL, Miller SD. Epitope spreading in immune-mediated diseases: implications for immunotherapy. Nat. Rev. Immunol. 2(2), 85-95 (2002).

6 Getts DR, Turley DM, Smith CE et al. Tolerance induced by apoptotic antigencoupled leukocytes is induced by PD- $\mathrm{L}^{+}$and IL-10-producing splenic macrophages and maintained by $\mathrm{T}$ regulatory cells. J. Immunol. 187(5), 2405-2417 (2011).

7 Getts DR, Martin AJ, Mccarthy DP et al. Microparticles bearing encephalitogenic peptides induce T-cell tolerance and ameliorate experimental autoimmune encephalomyelitis. Nat. Biotechnol. 30(12), 1217-1224 (2012).

8 Quintana FJ, Farez MF, Viglietta V et al. Antigen microarrays identify unique serum autoantibody signatures in clinical and pathologic subtypes of multiple sclerosis. Proc. Natl Acad. Sci. USA 105(48), 18889-18894 (2008).
9 Quintana FJ. The aryl hydrocarbon receptor: a molecular pathway for the environmental control of the immune response. Immunology 138(3), 183-189 (2013).

10 Apetoh L, Quintana FJ, Pot C et al. The aryl hydrocarbon receptor interacts with c-Maf to promote the differentiation of type 1 regulatory $\mathrm{T}$ cells induced by IL-27. Nat. Immunol. 11(9), 854-861 (2010).

11 Gandhi R, Kumar D, Burns EJ et al. Activation of the aryl hydrocarbon receptor induces human type 1 regulatory $\mathrm{T}$ cell-like and Foxp3(+) regulatory $\mathrm{T}$ cells. Nat. Immunol. 11(9), 846-853 (2010).

12 Quintana FJ, Basso AS, Iglesias AH et al. Control of T(reg) and $\mathrm{T}(\mathrm{H}) 17$ cell differentiation by the aryl hydrocarbon receptor. Nature 453, 65-71 (2008).

13 Quintana FJ, Jin H, Burns EJ et al. Aiolos promotes $\mathrm{T}(\mathrm{H}) 17$ differentiation by directly silencing Il2 expression. Nat. Immunol. 13(8), 770-777 (2012).

14 Quintana FJ, Murugaiyan G, Farez MF et al. An endogenous aryl hydrocarbon receptor ligand acts on dendritic cells and $\mathrm{T}$ cells to suppress experimental autoimmune encephalomyelitis. Proc. Natl Acad. Sci. USA 107(48), 20768-20773 (2010).

15 Wu HY, Quintana FJ, Da Cunha AP et al. In vivo induction of $\operatorname{Tr} 1$ cells via mucosal dendritic cells and AHR signaling. PLoS ONE 6(8), e23618 (2011).

16 Yeste A, Nadeau M, Burns EJ, Weiner HL, Quintana FJ. Nanoparticle-mediated codelivery of myelin antigen and a tolerogenic small molecule suppresses experimental autoimmune encephalomyelitis. Proc. Natl
Acad. Sci. USA 109(28), 11270-11275 (2012).

17 Hauben E, Gregori S, Draghici E et al. Activation of the aryl hydrocarbon receptor promotes allograft-specific tolerance through direct and dendritic cell-mediated effects on regulatory T cells. Blood 112(4), 1214-1222 (2008).

18 Mezrich JD, Fechner JH, Zhang X, Johnson BP, Burlingham WJ, Bradfield CA. An interaction between kynurenine and the aryl hydrocarbon receptor can generate regulatory T cells. J. Immunol. 185(6), 3190-3198 (2010).

19 Nguyen NT, Kimura A, Nakahama T et al. Aryl hydrocarbon receptor negatively regulates dendritic cell immunogenicity via a kynurenine-dependent mechanism. Proc. Natl Acad. Sci. USA 107(46), 19961-19966 (2010).

20 Nguyen LP, Bradfield CA. The search for endogenous activators of the aryl hydrocarbon receptor. Chem. Res. Toxicol. 21(1), 102-116 (2008).

21 Mellor AL, Munn DH. IDO expression by dendritic cells: tolerance and tryptophan catabolism. Nat. Rev. Immunol. 4(10), 762-774 (2004).

22 Opitz CA, Litzenburger UM, Sahm F et al. An endogenous tumour-promoting ligand of the human aryl hydrocarbon receptor. Nature 478(7368), 197-203 (2011).

23 Hall JA, Grainger JR, Spencer SP, Belkaid Y. The role of retinoic acid in tolerance and immunity. Immunity 35(1), 13-22 (2011).

24 Paciotti GF, Myer L, Weinreich D et al. Colloidal gold: a novel nanoparticle vector for tumor directed drug delivery. Drug Deliv. 11(3), 169-183 (2004). 